\title{
N=2 SUGRA BPS multi-center black holes and freudenthal triple systems
}

\author{
E. Torrente-Lujan ${ }^{1, a}$, J.J. Fernandez-Melgarejo² \\ ${ }^{1}$ IFT, Dept. of Physics, U. Murcia, Murcia, SPAIN. \\ ${ }^{2}$ Dept. of Physics, Harvard University, USA
}

\begin{abstract}
.
We present a detailed description of $N=2$ stationary BPS multicenter black hole solutions for quadratic prepotentials with an arbitrary number of centers and scalar fields making a systematic use of the algebraic properties of the matrix of second derivatives of the prepotential, $\mathcal{S}$, which in this case is a scalar-independent matrix. The anti-involution matrix $\mathcal{S}$ can be understood as a Freudenthal duality $\tilde{x}=\mathcal{S} x$. We show that this duality can be generalized to "Freudenthal transformations"

$$
x \rightarrow \lambda \exp (\theta \mathcal{S}) x=a x+b \tilde{x}
$$

under which the horizon area, ADM mass and intercenter distances scale up leaving constant the scalars at the fixed points. In the special case $\lambda=1$, " $\mathcal{S}$-rotations", the transformations leave invariant the solution. The standard Freudenthal duality can be written as $\tilde{x}=\exp \left(\frac{\pi}{2} \mathcal{S}\right) x$. We argue that these generalized transformations leave invariant not only the quadratic prepotential theories but also the general stringy extremal quartic form $\Delta_{4}$, $\Delta_{4}(x)=\Delta_{4}(\cos \theta x+\sin \theta \tilde{x})$ and therefore its entropy at lowest order.
\end{abstract}

\section{N2 D4 SUGRA and Special Kähler geometry}

The field content of the $N=2$ supergravity theory coupled to $n_{v}$ vector multiplets consists of ${ }^{1}$

$$
\left\{e_{\mu}{ }^{a}, A_{\mu}^{I}, z^{\alpha}, \psi_{\mu}^{r}, \lambda_{r}^{\alpha}\right\}
$$

with $\alpha=1, \ldots, n_{v}$, and $I=0, \ldots, n_{v}$. The theory also contains some hypermultiplets, which can be safely taken as constant or neglected (further details can be found in [1], whose notation and concepts we generally adopt). The bosonic $N=2$ action can be written as

$$
S=\int_{M(4 d)} R \star 1+\mathcal{G}_{\alpha \bar{\beta}} d z^{\alpha} \wedge \star d \bar{z}^{\bar{\beta}}+F^{I} \wedge G_{I}
$$

\footnotetext{
ae-mail: etl@um.es

${ }^{1}$ See [1] and references therein for notation fixing and a review of the topic.
} 
The fields $F^{I}, G_{I}$ are not independent: $F^{I}=d A^{I}$ and $G_{I}=a_{I J} F^{I}+b_{I J} \star F^{I}$ with scalar-dependent coefficients $a_{I J}$ and $b_{I J}$. Abelian charges with respect the $U(1)^{n_{v}+1}$ local symmetry of the theory are defined by means of the integrals of the gauge field strengths. The total charges are

$$
q \equiv\left(p^{I}, q_{I}\right) \equiv \frac{1}{2 \pi^{2}} \int_{S_{\infty}}\left(F^{I}, G_{I}\right) .
$$

The theory is defined, making use of the special geometry formalism, by some projective scalar coordinates $X^{I}$, as for example, 'special' projective coordinates $z^{\alpha} \equiv X^{\alpha} / X^{0}$. By introducing a covariantly holomorphic section of a symplectic bundle, $V$, we are able to arrange $2 n_{v}$ quantities that transform as a vector under symplectic transformations at any point of the manifold. $V$ has the following structure $V=V(z, \bar{z}) \equiv\left(V^{I}, V_{I}\right)$ and satisfies the following identities:

$$
\langle V \mid \bar{V}\rangle \equiv V^{t} \omega \bar{V} \equiv \bar{V}^{I} V_{I}-V^{I} \bar{V}_{I}=-i
$$

where $\omega$ is the symplectic form. ${ }^{2}$

The scalar kinetic term in the action can be written in terms of $V$ as $L_{s, k i n} \sim i\left\langle D^{\mu} \bar{V} \mid D_{\mu} V\right\rangle$ and the scalar metric is given by $\mathcal{G}_{\alpha \bar{\beta}}=\partial_{\alpha} \partial_{\bar{\beta}} \mathcal{K}$ where the Kähler potential $\mathcal{K}$ is defined by the relations $V=\exp (\mathcal{K} / 2) \Omega$ being $\Omega \equiv\left(X^{I}, F_{I}\right)$ a holomorphic section and

$$
e^{-\mathcal{K}}=i\left(\bar{X}^{I} F_{I}-X^{I} \bar{F}_{I}\right)=i\langle\Omega \mid \bar{\Omega}\rangle \text {. }
$$

The central charge $Z$ is

$$
Z\left(z^{\alpha}, q\right) \equiv\langle V \mid q\rangle=e^{\mathcal{K} / 2}\left(p^{I} F_{I}-q_{I} X^{I}\right)
$$

The embedding of the isometry group of the scalar manifold metric $\mathcal{G}_{\alpha \bar{\beta}}$, into the symplectic group fixes, through the Kähler potential $\mathcal{K}$, a functional relation between the lower and upper parts of $V$ and $\Omega[?$ ? $]$,

$$
\begin{aligned}
& F_{I}=F_{I}\left(X^{I}\right), \\
& V_{I}=V_{I}\left(V^{I}\right) .
\end{aligned}
$$

There always exists a symplectic frame under which the theory can be described in terms of a single holomorphic function, the prepotential $F(X)$. It is a second degree homogeneous function on the projective scalar coordinates $X^{I}$, such that $F_{I}(X)=\partial_{I} F(X)$. For simplicity, we will assume the existence of such prepotential along this study although the results will not depend on such existence. Using the notation $F_{I J}=\partial_{I} \partial_{J} F$, the lower and upper components of $\Omega$ are related by

$$
F_{I}=F_{I J} X^{J} \text {. }
$$

The lower and upper components of $V$ are related by a field dependent matrix $N_{I J}$, which is determined by the special geometry relations [2]

$$
\begin{aligned}
V_{I} & =N_{I J} V^{J}, \\
D_{\bar{\imath}} \bar{V}_{I} & =N_{I J} D_{\bar{l}} \bar{V}^{J} .
\end{aligned}
$$

\footnotetext{
${ }^{2}$ We choose a basis such that $\omega=\left(\begin{array}{cc}0 & -1_{n_{v}} \\ 1_{n_{v}} & 0\end{array}\right)$.
} 
The matrix $N$, which also fixes the vector couplings $\left(a_{I J}, b_{I J}\right)$ in the action, can be related to $F_{I J}$ [3] by

$$
N_{I J}=\bar{F}_{I J}+T_{I} T_{J},
$$

where the quantities $T_{I}$ are proportional to the projector of the graviphoton, whose flux defines the $N=2$ central charge [3]. For our purposes, it is convenient to write the relation between $N_{I J}$ and $F_{I J}$ as

$$
\begin{aligned}
N_{I J} & \equiv F_{I J}+N_{I J}^{\perp} \\
& =F_{I J}-2 i \operatorname{Im}\left(F_{I J}\right)+2 i \frac{\operatorname{Im}\left(F_{I K}\right) L^{K} \operatorname{Im}\left(F_{J Q}\right) L^{Q}}{L^{P} \operatorname{Im}\left(F_{P Q}\right) L^{Q}},
\end{aligned}
$$

where we have decomposed the matrix $N_{I J}$ into "longitudinal" (the $F_{I J}$ themselves) and "transversal" parts $\left(N_{I J}^{\perp}\right)$. The perpendicular term (defined by the expression above) annihilates $L^{I}$, or any multiple of it,

$$
N_{I J}^{\perp}\left(\alpha L^{J}\right)=0 .
$$

From this, (10) can be written as

$$
\begin{aligned}
V_{I} & =N_{I J} L^{J}=\left(F_{I J}+N_{I J}^{\perp}\right) L^{J} \\
& =F_{I J} L^{J} .
\end{aligned}
$$

Thus, the upper and lower components of $V$ and $\Omega$ are connected by the same matrix $F_{I J}$.

The existence of functional dependencies among the upper and lower components of the vectors $V$ or $\Omega$ imply further relations between their respective real and imaginary parts. They are related by symplectic matrices $\mathcal{S}(N), \mathcal{S}(F) \in S p\left(2 n_{v}+2, \mathbb{R}\right)$ which are respectively associated to the quantities $N_{I J}, F_{I J}$ as follows:

$$
\begin{aligned}
& \operatorname{Re}(\Omega)=\mathcal{S}(F) \operatorname{Im}(\Omega), \\
& \operatorname{Re}(V)=\mathcal{S}(N) \operatorname{Im}(V)=\mathcal{S}(F) \operatorname{Im}(V) .
\end{aligned}
$$

The last expression is obtained by means of the relation (15). These same relations (16)-(17) are valid for any complex multiple of $\Omega$ or $V$. It is straightforward to show, for example, that for any $\lambda \in \mathbb{C}$, we have

$$
\operatorname{Re}(\lambda V)=\mathcal{S}(N) \operatorname{Im}(\lambda V)=\mathcal{S}(F) \operatorname{Im}(\lambda V) .
$$

The form of the matrices $\mathcal{S}(F), \mathcal{S}(N)$ is explicitly given in [1].

In $N=2$ theories, $\mathcal{S}(N)$ always exhibits a moduli dependence [4]. However, this is not the case for $\mathcal{S}(F)$. We will focus in this work on the particular case of theories with quadratic prepotentials,

$$
F(X)=\frac{1}{2} F_{I J} X^{I} X^{J}
$$

where $F_{I J}$ is a complex, constant, symmetric matrix. Then, the corresponding matrix $\mathcal{S}(F)$ is a fieldindependent, constant matrix. We can assume that $\operatorname{Re}\left(F_{I J}\right)=0$ and $\operatorname{Im}\left(F_{I J}\right)$ is negative definite. In what follows, we will use the notation $\mathcal{S} \equiv \mathcal{S}(F)$. The condition $e^{-\mathcal{K}}>0$ and the expression (5) implies a restriction on the prepotential. 


\subsection{General supersymmetric stationary solutions}

The most general stationary (time independent) 4-dimensional metric compatible with supersymmetry can be written in the IWP form [5-7],

$$
d s^{2}=e^{2 U}(d t+\omega)^{2}-e^{-2 U} d \mathbf{x}^{2}
$$

Supersymmetric $N=2$ supergravity solutions can be constructed systematically following well established methods $[8,9]$. In this section we will closely follow the notation of ref.[9]. The 1-form $\omega$ and the function $e^{-2 U}$ are related in these theories to the Kähler potential and connection, $\mathcal{K}, Q$ [7]. We demand asymptotic flatness, $e^{-2 U} \rightarrow 1$ together with $\omega \rightarrow 0$ for $|\mathbf{x}| \rightarrow \infty$. BPS field equation solutions for the action above (for example, quantities that appear in the metric, as $e^{-2 U}$ or $\omega$ ) can be written in terms of the following real symplectic vectors $\mathcal{R}$ and $\mathcal{I}$

$$
\mathcal{R}=\frac{1}{\sqrt{2}} \operatorname{Re}\left(\frac{V}{X}\right), \mathcal{I}=\frac{1}{\sqrt{2}} \operatorname{Im}\left(\frac{V}{X}\right) .
$$

$X$ is an arbitrary complex function of space coordinates such that $1 / X$ is harmonic. The $2 n_{v}+2$ components of $\mathcal{I}$ and $\mathcal{R}$ are real harmonic functions in $\mathbb{R}^{3}$. There is an algebraic relation between $\mathcal{R}$ and $\mathcal{I}$ and the solutions can be written in terms only of the vector $\mathcal{I}$. By making use of (16)-(18), we can write the equation

$$
\mathcal{R}=\mathcal{S I}
$$

In practice, specific solutions are determined by giving a particular, suitable, ansatz for the symplectic vector $\mathcal{I}$ as a function of the spacetime coordinates.

Using these symplectic vectors we rewrite the only independent metric component as

$$
e^{-2 U}=e^{-\mathcal{K}}=\frac{1}{2|X|^{2}}=\langle\mathcal{R} \mid \mathcal{I}\rangle=\langle\mathcal{S I} \mid \mathcal{I}\rangle
$$

Similarly, the time independent 3-dimensional 1-form $\omega=\omega_{i} d x^{i}$ satisfies the equation

$$
d \omega=2\left\langle\mathcal{I} \mid \star_{3} d \mathcal{I}\right\rangle,
$$

where $\star_{3}$ is the Hodge dual on flat $\mathbb{R}^{3}$, together with the integrability condition

$$
\langle\mathcal{I} \mid \Delta \mathcal{I}\rangle=0 \text {. }
$$

The asymptotic flatness condition implies

$$
\left\langle\mathcal{R}_{\infty} \mid \mathcal{I}_{\infty}\right\rangle=\left\langle S \mathcal{I}_{\infty} \mid \mathcal{I}_{\infty}\right\rangle=1
$$

The gauge field equations of motion and Bianchi identities can be directly solved in terms of spatially dependent harmonic functions [9]. The modulus of the central charge function defined in (6) can be written, taking into account (23), as

$$
|Z(q)|^{2} e^{-2 U}=|\langle\mathcal{R} \mid q\rangle|^{2}+|\langle\mathcal{I} \mid q\rangle|^{2} .
$$

At spatial infinity, by assuming the asymptotic flatness condition (26), we arrive to

$$
\left|Z_{\infty}(q)\right|^{2}=\left|\left\langle\mathcal{R}_{\infty} \mid q\right\rangle\right|^{2}+\left|\left\langle\mathcal{I}_{\infty} \mid q\right\rangle\right|^{2} .
$$


The, assumed time independent, $n_{v}$ complex scalar fields $z^{\alpha}$ solutions to the field equations, are given in this formalism by

$$
z^{\alpha}=\frac{\Omega^{\alpha}}{\Omega^{0}}=\frac{V^{\alpha} / X}{V^{0} / X}=\frac{\mathcal{R}^{\alpha}+i \mathcal{I}^{\alpha}}{\mathcal{R}^{0}+i \mathcal{I}^{0}} .
$$

This is, in general, a formal expression as the $\mathcal{I}$ or $\mathcal{R}$ quantities may be scalar dependent. ${ }^{3}$ These scalar fields can, in principle, take any values $\left(z_{\infty}\right)$ at infinity. These values will appear as free parameters in the ansatz that we give for $\mathcal{I}$. Nevertheless, according to the attractor mechanism, the moduli adjust themselves at some fixed points.

We are interested in this work in extremal, single- or multi-center black hole-type solutions determined by an $\mathcal{I}$ ansatz with point-like singularities of the form

$$
\mathcal{I}=\mathcal{I}_{\infty}+\sum_{a} \frac{q_{a}}{\left|\mathbf{x}-\mathbf{x}_{a}\right|},
$$

where $a=1, \ldots, n_{a}$ being the number $n_{a}$ arbitrary and $q_{a}=\left(p_{a}{ }^{I}, q_{a I}\right)$ and $\mathcal{I}_{\infty}$ real, constant, symplectic vectors.

For this kind of solutions, the quantities $\mathcal{I}_{\infty}$ are related to the values at infinity of the moduli while the "charge" vectors $q_{a}$ are related to their values at the fixed points. The fixed values of the scalars, $z(\mathbf{x}) \rightarrow z\left(\mathbf{x}_{a}\right)=z_{f}^{a}$, are the solutions of the following attractor equations $[2,10,11]$ :

$$
q^{a}=\operatorname{Re}\left(2 i \bar{Z}\left(z_{f}^{a}\right) V\left(z_{f}^{a}\right)\right)
$$

The prepotential performs its influence throughout $V$ and $Z$ (cf. (6)). The scalar attractor values are independent of their asymptotic values and only depend on the discrete charges $z_{f}^{a}=z_{f}^{a}\left(q_{a}\right)$.

\section{The stabilization matrix, its adjoint and the attractor equations}

It can be shown by explicit computation that the real symplectic matrices $\mathcal{S}_{N}, \mathcal{S} \equiv \mathcal{S}_{F} \in S p\left(2 n_{v}+2, \mathbb{R}\right)$ defined by (16)-(17), whose explicit expressions are (??), satisfy the relations (see also [3] )

$$
\mathcal{S}_{N}^{2}=\mathcal{S}_{F}^{2}=-1 \text {. }
$$

We define the projector operators $P_{ \pm}=\frac{1 \pm i S}{2}$. They satisfy the following straightforward properties

$$
\begin{aligned}
P_{ \pm}^{2} & =P_{ \pm},\left(P_{ \pm}\right)^{*}=P_{\mp} . \\
S P_{ \pm} & =\mp i P_{ \pm},
\end{aligned}
$$

For $X, Y$ arbitrary real vectors, we have

$$
P_{ \pm} X=P_{ \pm} Y \quad \Rightarrow \quad X=Y .
$$

$P_{ \pm}$are the projectors on the eigenspaces (of equal dimension) of the matrix $\mathcal{S}$. $W$ can be decomposed into eigenspaces of the matrix $\mathcal{S}$ : $W=W^{+} \oplus W^{-}$, where $W^{ \pm} \equiv P_{ \pm} W$. For an arbitrary function of $\mathcal{S}$, $f(\mathcal{S})$, necessarily a linear function of it, $f(\mathcal{S}) \equiv a+b \mathcal{S} \equiv \lambda \exp (\theta \mathcal{S})$, we have

$$
f(\mathcal{S}) P_{ \pm}=f(\mp i) P_{ \pm} .
$$

\footnotetext{
${ }^{3}$ Even for a scalar independent ansatz $\mathcal{I}$, the matrix $\mathcal{S}$ is, in general, scalar dependent.
} 
Complex conjugation interchanges $W^{+}$and $W^{-}$subspaces, both subspaces are isomorphic to each other and of dimension $n_{v}+1$.

For arbitrary $\lambda \in \mathbb{C}$ and $V \in W$, for which there is a relation between its real and imaginary parts of the form $\operatorname{Re}(V)=\mathcal{S} \operatorname{Im}(V)$, we have

$$
\lambda V=\operatorname{Re}(\lambda V)+i \operatorname{Im}(\lambda V)=2 i \mathrm{P}_{-} \operatorname{Im}(\lambda V) .
$$

Thus, the full vector $V$ can be reconstructed applying one of the projectors either from its real or imaginary part. Such vectors are fully contained in the subspace $W^{-}$or, equivalently, they are eigenvectors of $\mathcal{S}$

$$
\mathcal{S} V=2 i \mathrm{SP}_{-} \operatorname{Im}(V)=2 \mathrm{P}_{-} \operatorname{Im}(V)=i V .
$$

We define the adjoint operator $\mathcal{S}^{\dagger}$ of the matrix $\mathcal{S}$, with respect to the symplectic bilinear product so that, for any vectors $A, B \in W,\langle\mathcal{S} A \mid B\rangle=\left\langle A \mid \mathcal{S}^{\dagger} B\right\rangle . \mathcal{S}^{\dagger}$ is given by $\mathcal{S}^{\dagger}=-\omega \mathcal{S}^{t} \omega$. Under the assumption of a symmetric $F_{I J}$ matrix, it is given by

$$
\mathcal{S}^{\dagger}=-\mathcal{S} \text {. }
$$

$\mathcal{S}$ defines an (almost) complex structure on the symplectic space. This complex structure preserves the symplectic bilinear form, the matrix $\mathcal{S}$ is an isometry of the symplectic space,

$$
\langle\mathcal{S} X \mid \mathcal{S} Y\rangle=\langle X \mid Y\rangle \text {. }
$$

From (38), we see that $\mathcal{S}$ is an element of the symplectic Lie algebra $\mathfrak{s p}\left(2 n_{v}+2\right)$. Moreover, the bilinear form defined by $g(X, Y) \equiv\langle\mathcal{S} X \mid Y\rangle$ is symmetric. $^{4}$

In general, the matrices $\mathcal{S}_{N}, \mathcal{S}_{F}$ are scalar dependent. Only one of them, $\mathcal{S}_{F}$, is constant, in the case of quadratic prepotentials. Let us write $\mathcal{S}_{N}^{f} \equiv \mathcal{S}_{N}\left(z_{f}\right) \mathcal{S}_{F}^{f} \equiv \mathcal{S}_{F}\left(z_{f}\right)$ for the matrices evaluated at (anyone of) the fixed points. Let us use the sub/superindex $f$ to denote any quantity at the fixed points. We arrive to (using (17) and (18)

$$
\mathcal{S}_{N}^{f} q^{a}=\mathcal{S}_{N}^{f} \operatorname{Re}\left(2 i \bar{Z}^{f} V^{f}\right)=\mathcal{S}_{F}^{f} \operatorname{Re}\left(2 i \bar{Z}^{f} V^{f}\right)=\mathcal{S} q^{a} .
$$

The attractor equations can be written yet in another alternative way. By using (36) and (31), we can write

$$
i \bar{Z}^{f} V^{f}=2 \mathrm{P}_{-} i \bar{Z}^{f} V^{f}=\mathrm{P}_{-} q .
$$

That is, the attractor equations simply equalize (a multiple of) the vector $V$ (which, as we have seen above, lies in the subspace $W^{-}$) with the part of the charge vector which lies in $W^{-}$.

From (41) and taking $\langle V \mid \bar{V}\rangle=-i$, we arrive to

$$
\left|Z^{f}\right|^{2}=\frac{1}{2}\langle\mathcal{S} q \mid q\rangle
$$

Moreover the consistency condition $e^{-\mathcal{K}}>0$ can be written as (see (5))

$$
\begin{aligned}
e^{-\mathcal{K}} & =i\langle\Omega \mid \bar{\Omega}\rangle=2\langle\operatorname{Re}(\Omega) \mid \operatorname{Im}(\Omega)\rangle \\
& =2\langle\mathcal{S} \operatorname{Im}(\Omega) \mid \operatorname{Im}(\Omega)\rangle>0 .
\end{aligned}
$$

This condition is not automatically satisfied as the symmetric quadratic form $g$ is indefinite. ${ }^{5}$

\footnotetext{
${ }^{4}$ The quadratic form $g(X, X)$ is also known as the " $\mathcal{I}_{2}(X)$ " in the literature. The corresponding quartic invariant in this case can be written as $\mathcal{I}_{4}(X, \ldots)=\langle X \mid X\rangle^{2}$.

${ }^{5}$ The matrix $\omega \mathcal{S}$ it has an even number of negative eigenvalues as $\operatorname{det} \omega \mathcal{S}=(-1)^{2 n_{v}+2}=1$. The signature of $g$ is $\left(2 n_{v}, 2\right)$.
} 


\section{1 $\mathcal{S}$ transformations and Freudenthal duality}

Let us consider " $\mathcal{S}$-transformations" of the type

$$
X \rightarrow X^{\prime}=f(\mathcal{S}) X,
$$

where $f$ is an arbitrary function. $f(\mathcal{S})$ can be written with full generality as a linear expression

$$
f(\mathcal{S}) \equiv a+b \mathcal{S}
$$

or in "polar form"

$$
f(\mathcal{S}) \equiv \lambda \exp (\theta \mathcal{S})
$$

where $a, b$ or $\lambda, \theta$ are real parameters. The adjoint is $f(\mathcal{S})^{\dagger}=f\left(\mathcal{S}^{\dagger}\right)=f(-\mathcal{S}), f^{\dagger} f=a^{2}+b^{2}=\lambda^{2}$. Under these transformations $f=f(\mathcal{S})$ the symplectic and $g$ bilinear products become scaled:

$$
\begin{aligned}
\left\langle X^{\prime} \mid Y^{\prime}\right\rangle & =\lambda^{2}\langle X \mid Y\rangle, \\
\left\langle\mathcal{S} X^{\prime} \mid Y^{\prime}\right\rangle & =\lambda^{2}\langle\mathcal{S} X \mid Y\rangle .
\end{aligned}
$$

If $\lambda=1$ both products are invariant under the Abelian $U(1)$-like group of transformations

$$
U_{F}(\theta)=\exp \theta \mathcal{S},
$$

the "S-rotations". Any physical quantity (entropy, ADM Mass, scalars at fixed points, intercenter distances, etc.) written in terms of these products (as it will clearly appear in the next sections) will automatically be scaled under the general transformations or invariant under the rotations.

On the other hand it can be easily checked that the "degenerate" Freudenthal duality transformation [12-15]. is given in our case by the the anti-involutive transformation

$$
\tilde{X}=\omega \frac{\partial g(X, X)}{\partial X}=\mathcal{S} X,
$$

with $\tilde{\tilde{X}}=-X$.

The Freudenthal duality corresponds to a particular $\mathcal{S}$-transformation, a $\mathcal{S}$-rotation of the type

$$
f(\mathcal{S})=\exp ((\pi / 2) \mathcal{S}) .
$$

Invariance of quantities as ADM mass and entropy under Freudenthal duality is a special case of a more general behavior of the solutions under the (Abelian) group of general $\mathcal{S}$-transformations. A general $\mathcal{S}$-transformations can be written in terms of Freudenthal duality as

$$
X \rightarrow \tilde{X}(a, b)=a X+b \tilde{X},
$$

or,

$$
\tilde{X}(\lambda, \theta)=\lambda \cos \theta X+\lambda \sin \theta \tilde{X} .
$$

\section{Complete solutions for quadratic prepotentials}

\subsection{Behavior of the scalar field solutions}

In the previous section we have obtained some general results without assuming a specific form for the solutions $\mathcal{I}$. In this section we will make use of the ansatz (30) for theories with quadratic prepotentials to obtain a full characterization of the solutions. 
Let us insert the ansatz (30) into the general expression for the complex scalars, (29). The values for the time independent $n_{v}$ complex scalars, solutions to the field equations, are explicitly given by

$$
z^{\alpha}(\mathbf{x})=\frac{\left(\mathrm{P}_{-} \mathcal{I}\right)^{\alpha}}{\left(\mathrm{P}_{-} \mathcal{I}\right)^{0}}
$$

This expression interpolates between the values at the fixed points and at infinity. It can be written as $\left(c_{\infty}^{\alpha}(\mathbf{x})\right.$ and $c_{a}^{\alpha}(\mathbf{x})$ are spatial dependent complex functions $)$

$$
z^{\alpha}(\mathbf{x})=c_{\infty}^{\alpha}(\mathbf{x}) z_{\infty}^{\alpha}+\sum_{a} c_{a}^{\alpha}(\mathbf{x}) z_{a, f}^{\alpha}
$$

The scalar charges $\Sigma^{\alpha}$ associated to the scalar fields can be defined by the asymptotic series

$$
z^{\alpha}(r \rightarrow \infty)=z_{\infty}^{\alpha}+\frac{\Sigma^{\alpha}}{r}+O\left(\frac{1}{r^{2}}\right)
$$

Expanding (??), we have

$$
z^{\alpha}(r \rightarrow \infty)=z_{\infty}^{\alpha}+\frac{r_{0}\left(z_{f}^{\alpha}(Q)-z_{\infty}^{\alpha}\right)}{r}+O\left(\frac{1}{r^{2}}\right),
$$

and thus the scalar charges are given by

$$
\Sigma^{\alpha}=r_{0}\left(z_{f}^{\alpha}(Q)-z_{\infty}^{\alpha}\right) .
$$

Hence, the scalar charges are fixed in terms of the charge vectors and the asymptotic moduli. In the special case of a single center solution, the expression (54) is in agreement with the well known fact that the scalar charges vanish for double extremal black holes. In the multicenter case, from this formula we infer a similar result: the scalar charges vanish if

$$
z_{\infty}^{\alpha}=z_{f}^{\alpha}(Q)
$$

Obviously, this does not mean that the scalar fields are constant in all space. Therefore, the conditions (55) could be considered a convenient generalization of double extremal solutions in the multicenter case. Taking into account the considerations of the previous section, (??), a possible vector $\mathcal{I}_{\infty}$ corresponding to this solution would be of the form

$$
\mathcal{I}_{\infty}= \pm \frac{Q}{\sqrt{\langle\mathcal{S Q} \mid Q\rangle}},
$$

and the scalar fields would be parametrized at any point of the space by

$$
z^{\alpha}(\mathbf{x})=c_{\infty}^{\alpha}(\mathbf{x}) z_{f}^{\alpha}(Q)+\sum_{a} c_{a}^{\alpha}(\mathbf{x}) z_{f}^{\alpha}\left(q_{a}\right)
$$

\subsection{Intercenter distances and $\mathcal{S}$-transformations}

The charge interdistances are restricted, from the 1-form $\omega$ condition of integrability [9], we have (for any charge center $q_{b}$ )

$$
\left\langle\mathcal{I}_{\infty} \mid q_{b}\right\rangle+\sum_{a} \frac{\left\langle q_{a} \mid q_{b}\right\rangle}{r_{a b}}=0,
$$


where $r_{a b}=\left|\mathbf{x}_{a}-\mathbf{x}_{b}\right|$. The solutions for this set of equations give the possible values of the center positions.

Let us study the effect of $\mathcal{S}$-transformations on the intercenter distances for transformed $\mathcal{I}_{\infty}$ and charge symplectic vectors. The vector $\mathcal{I}_{\infty}$ is constrained by the asymptotic flatness condition to a unit fixed $g$-norm, $1=\left\langle\mathcal{S} \mathcal{I}_{\infty} \mid \mathcal{I}_{\infty}\right\rangle$. We consider therefore set of transformations of the type

$$
\begin{aligned}
\mathcal{I}_{\infty} \rightarrow \tilde{I}_{\infty}(\theta) & =\exp (\theta \mathcal{S}) \mathcal{I}_{\infty}, \\
q_{a} \rightarrow \tilde{q}_{a}(\lambda, \theta) & =\lambda \exp (\theta \mathcal{S}) q_{a} .
\end{aligned}
$$

Under these transformations the equations (58) become

$$
\lambda\left\langle\mathcal{I}_{\infty} \mid q_{b}\right\rangle+\lambda^{2} \sum_{a} \frac{\left\langle q_{a} \mid q_{b}\right\rangle}{\tilde{r}_{a b}}=0 .
$$

Then

$$
r_{a b} \rightarrow \tilde{r}_{a b}=\lambda r_{a b}
$$

the intercenter distances scale (remain invariant) under general $\mathcal{S}$-transformations $(\mathcal{S}$-rotations or Freudenthal dualities) of the charge and $\mathcal{I}_{\infty}$ vectors.

Let us see the consequence of the integrability equations for a double extremal two center configuration. In this case, if $\mathcal{I}_{\infty}=\lambda Q$, we have

$$
\begin{aligned}
0 & =\lambda\left\langle Q \mid q_{2}\right\rangle+\frac{\left\langle q_{1} \mid q_{2}\right\rangle}{r_{12}} \\
& =\left\langle q_{1} \mid q_{2}\right\rangle\left(\lambda+\frac{1}{r_{12}}\right) .
\end{aligned}
$$

If we compare this equation with (56), we conclude that, if $\left\langle q_{1} \mid q_{2}\right\rangle \neq 0$, we have to choose the negative sign there and the double extremal intercenter distance is given by

$$
\left.r_{12}^{2}\right|_{\text {double ext. }}=\langle\mathcal{S} Q \mid Q\rangle .
$$

In the case $\left\langle q_{1} \mid q_{2}\right\rangle=0$ the intercenter distance is not restristed by the compatibility equation Eq.(58).

\subsection{Near horizon and infinity geometry}

The metric has the form given by (20), with the asymptotic flatness conditions $-g_{r r}=\left\langle\mathcal{R}_{\infty} \mid \mathcal{I}_{\infty}\right\rangle=1$ and $\omega(|\mathbf{x}| \rightarrow \infty) \rightarrow 0$. For point-like sources, as those represented by the ansatz (30), the compatibility equation (25) takes the form [9]

$$
N \equiv \sum_{a}\left\langle\mathcal{I}_{\infty} \mid q_{a}\right\rangle=\left\langle\mathcal{I}_{\infty} \mid Q\right\rangle=0
$$

An explicit computation of the total field strength shows that (64) is equivalent to the requirement of absence of NUT charges: only after imposing the condition $N=0$, the overall integral of the $\left(F^{I}, G_{I}\right)$ field strengths at infinity is equal to $Q=\sum q_{a}$. Another consequence of the condition $N=0$, which can be checked by direct computation from (24), is that the 1-form $\omega$ takes the same value at any of the horizons of the centers that make up the multicenter black hole. This value is also equal to its value at spacial infinity, which can be taken to be zero. 
We can write, using (22) and the ansatz (30), the expression

$$
\begin{aligned}
-g_{r r}=\langle\mathcal{R} \mid \mathcal{I}\rangle & = \\
1+2 & \sum_{b} \frac{\left\langle\mathcal{S} I_{\infty} \mid q_{b}\right\rangle}{\left|\mathbf{x}-\mathbf{x}_{b}\right|}+\sum_{a, b} \frac{\left\langle\mathcal{S} q_{a} \mid q_{b}\right\rangle}{\left|\mathbf{x}-\mathbf{x}_{a}\right|\left|\mathbf{x}-\mathbf{x}_{b}\right|},
\end{aligned}
$$

where we have used the property $\mathcal{S}^{\dagger}=-\mathcal{S}$ and the asymptotic flatness condition $\left\langle\mathcal{S} \mathcal{I}_{\infty} \mid \mathcal{I}_{\infty}\right\rangle=1$. We introduce now the quantities

$$
\begin{aligned}
M_{a} & \equiv\left\langle\mathcal{S} I_{\infty} \mid q_{a}\right\rangle, \\
A_{a b} & \equiv\left\langle\mathcal{S} q_{a} \mid q_{b}\right\rangle,
\end{aligned}
$$

where $A_{a b}$ is symmetric in its indices due to the property (??). The metric element is then

$$
-g_{r r}=1+2 \sum_{a} \frac{M_{a}}{\left|\mathbf{x}-\mathbf{x}_{a}\right|}+\sum_{a, b} \frac{A_{a b}}{\left|\mathbf{x}-\mathbf{x}_{a}\right|\left|\mathbf{x}-\mathbf{x}_{b}\right|}
$$

If the metric element (68) describes a black hole, then the right part should be kept always positive and finite for any finite $|\mathbf{x}|{ }^{6}$ A sufficient condition for its positivity is, for example, that the mass-like $M_{a}$ and area-like $A_{a b}$ parameters are all positive.

\section{Behavior at fixed points and at infinity}

At spatial infinity $|\mathbf{x}| \rightarrow \infty, 1 /\left|\mathbf{x}-\mathbf{x}_{a}\right| \rightarrow 1 / r$, the metric element (68) becomes spherically symmetric:

$$
-g_{r r}=1+\frac{2 \sum_{a} M_{a}}{r}+\frac{\sum_{a b} A_{a b}}{r^{2}}+O\left(\frac{1}{r^{3}}\right) .
$$

Then

$$
\begin{aligned}
M_{A D M} & =\sum_{a} M_{a}=\left\langle\mathcal{S} I_{\infty} \mid Q\right\rangle, \\
A_{\infty} & =\sum_{a b} A_{a b}=\langle\mathcal{S} Q \mid Q\rangle .
\end{aligned}
$$

The central charge at infinity, (28), becomes then

$$
\begin{aligned}
\left|Z_{\infty}\right|^{2} & =\left|\left\langle\mathrm{P}_{+} \mathcal{I}_{\infty} \mid Q\right\rangle\right|^{2}=|N+i M|^{2} \\
& =M_{A D M}^{2}+N^{2}
\end{aligned}
$$

where $N$ is defined by (64). The compatibility condition $N=0$ is equivalent to the saturation of a BPS condition

$$
\left|Z_{\infty}\right|^{2}=M_{A D M}^{2}=\left|\left\langle S I_{\infty} \mid Q\right\rangle\right|^{2}
$$

Let us suppose a configuration with null scalar charges. In this case $\mathcal{I}_{\infty}= \pm \lambda Q, \lambda=$ $1 / \sqrt{\langle\mathcal{S} Q \mid Q\rangle}$. We have $M_{A D M}= \pm / \lambda$, the positivity of $M_{A D M}$ obliges us to choose the positive sign. For a two center case this in turn implies $\left\langle q_{1} \mid q_{2}\right\rangle=0$ and $r_{12}$ unrestricted (see section (3.2)).

\footnotetext{
${ }^{6}$ Consider, for example, that $-g_{r r} \sim e^{-\mathcal{K}}>0$.
} 
Such $\mathcal{I}_{\infty}$ trivially satisfies the absence of NUT charge $(N=0)$ condition, and for it $z_{\infty}^{\alpha}=z_{f}(Q)$. This configuration can be considered a multicenter generalization of the double extremal solutions.

For $\mathbf{x} \rightarrow \mathbf{x}_{a}$ the metric element given by (68) becomes spherically symmetric. Moreover, it can be shown that, by fixing additive integration constants, we can take $\omega_{a}=\omega\left(\mathbf{x} \rightarrow \mathbf{x}_{a}\right)=0$ at the same time that $\omega_{\infty}=\omega(\mathbf{x} \rightarrow \infty)=0$. As a consequence, the metric at any of the horizon components with charge $q_{a}$ approaches an $A d S_{2} \times S^{2}$ metric of the form

$$
d s^{2}=\frac{r^{2}}{\left\langle\mathcal{S} q_{a} \mid q_{a}\right\rangle} d t^{2}-\frac{\left\langle\mathcal{S} q_{a} \mid q_{a}\right\rangle}{r^{2}} d \mathbf{x}^{2} .
$$

This is a Robinson-Bertotti-like metric. The Robertson-Bertotti-like mass parameter $M_{R B}$ is given by

$$
M_{R B, a}^{2}=\left\langle\mathcal{S} q_{a} \mid q_{a}\right\rangle,
$$

this is a charge extremal condition impliying the positivity of the charge products: $\left\langle\mathcal{S} q_{a} \mid q_{a}\right\rangle>0 .{ }^{7}$

Then, the near horizon geometry is completely determined in terms of the individual horizon areas $S_{h, a}=\left\langle\mathcal{S} q_{a} \mid q_{a}\right\rangle$. The horizon area $S_{h}$ is the sum of the areas of its disconnected parts

$$
S_{h}=\sum_{a} S_{h, a}=\sum_{a}\left\langle\mathcal{S} q_{a} \mid q_{a}\right\rangle=2 \sum_{a}\left|Z_{f, a}\right|^{2}
$$

This expression can be compared with the area corresponding to a single center black hole with the same total charge $Q=\sum_{a} q_{a}$, which is given by $S_{h}(q=Q)=\langle\mathcal{S} Q \mid Q\rangle$.

Let us finally remark that under $\mathcal{S}$-transformations $\tilde{I}_{\infty} \rightarrow \tilde{\mathcal{I}}_{\infty}(\theta), q_{a} \rightarrow \tilde{q}_{a}(\lambda, \theta)$ the $A D M$ mass and the horizon areas scale as

$$
\begin{aligned}
\tilde{M}_{A D M} & =\lambda M_{A D M}, \\
\tilde{S_{h}} & =\lambda^{2} S_{h} .
\end{aligned}
$$

Under the same transformations, the scalars at the fixed points and at infinity remain invariant whereas the intercenter distances $r_{a b}$ scale as (62).

\section{Freudenthal duals and charge vector expansions}

Any real symplectic vector $X$ can be expanded as

$$
X=2 \operatorname{Im}\left(Z(X) \bar{V}+g^{\alpha \bar{\beta}} D_{\alpha} Z(X) \bar{D}_{\bar{\beta}} \bar{V}\right)
$$

with $Z(X)=\langle V \mid X\rangle$. The existence and properties of such expansions are based on the symplectic properties of $V$ and its derivatives as well as on the existence of a anti-involution $\mathcal{S}(N)$ for which $\mathcal{S}(N) V=i V$ and $\mathcal{S}(N) D_{\alpha} V=i D_{\alpha} V .^{8}$

We will define here alternative expansions using the properties of the matrix $\mathcal{S} \equiv \mathcal{S}(F)$. As we have seen before, the projectors $P_{ \pm}$split the $\left(2 n_{v}+2\right)$-dimensional space $W$ into two $\left(n_{v}+1\right)$ dimensional eigenspaces

$$
W=W^{+} \oplus W^{-},
$$

\footnotetext{
${ }^{7}$ The positivity of these quantities implies diverse restrictions as the quadratic form $g(X, Y)=\langle\mathcal{S} X \mid Y\rangle$ is undefinite with a signature including an even number of negative signs.

${ }^{8}$ See for example Section 2.2.2 in [16] and references therein.
} 
in which the eigenvectors of $\mathcal{S}$ (therefore eigenvectors of general $\mathcal{S}$-transformations) with eigenvalues $\pm i$, respectively, lie ( $c f$. Section 3 ).

Given a set of generic real charge vectors $\left(q_{1}, \ldots, q_{n}\right)$, the sets $\left(\mathrm{P}_{+} q_{a}\right)$, respectively $\left(\mathrm{P}_{-} q_{a}\right)$, possibly completed with additional suitable vectors, can be considered a basis for the eigenspaces $W^{+}$, respectively $W^{-}$. Let us consider the $W$ subspace $B\left(q_{n}\right)$ generated by eigenvectors of the matrix $\mathcal{S}$ associated to center charges, directly of the complex form

$$
B\left(q_{n}\right) \equiv \operatorname{Span}\left(P_{ \pm} q_{1}, \ldots, P_{ \pm} q_{n}\right),
$$

or, equivalently, in the real basis formed by charge vectors and their Freudenthal duals $\tilde{q}_{i}=\mathcal{S} q_{i}$

$$
B\left(q_{n}\right) \equiv \operatorname{Span}\left(q_{1}, \ldots, q_{n}, \mathcal{S} q_{1}, \ldots, \mathcal{S} q_{n}\right) .
$$

In particular, we can consider the subspace $B\left(q_{n a}\right)$ generated by the $n_{a}$ pairs $\left(q_{a}, \mathcal{S} q_{a}\right)$ of center charges, whose dimension is, in general, $\operatorname{dim} B\left(q_{n a}\right) \leq 2 n_{a}$. The dimension of the orthogonal complement to this space, $B\left(q_{n a}\right)^{\perp}$, i.e. those vectors $s$ such that $\langle q \mid s\rangle=\langle\mathcal{S} q \mid s\rangle=0$ is, generically, $\operatorname{dim} B\left(q_{n a}\right)^{\perp}=$ $2\left(n_{v}-n_{a}\right)+2 .^{9}$ This dimension is zero for one scalar, one center black holes $\left(n_{v}=0, n_{a}=1\right)$. The set of vectors $\left(q_{a}, \mathcal{S} q_{a}\right)$ may form themselves a (maybe overcomplete) basis for the $\left(2 n_{v}+2\right)$ symplectic space. Otherwise, they can be extended with as many other vectors $\left(s_{i}\right)$ as necessary to complete such basis. Any real symplectic vector of interest $\left(e . g . I_{\infty}\right)$ can be conveniently expanded as

$$
X=2 \operatorname{Re} \alpha^{a} \mathrm{P}_{+} q_{a}+2 \operatorname{Re} \gamma^{i} \mathrm{P}_{+} s_{i},
$$

where $\alpha^{a}, \gamma^{i}$ are complex parameters or, equivalently, as

$$
X=\alpha^{a} q_{a}+\tilde{\alpha}^{a} \mathcal{S} q_{a}+\gamma^{i} s_{i}+\tilde{\gamma}^{i} \mathcal{S} s_{i}
$$

where $\alpha^{a}, \tilde{\alpha}^{a}, \gamma^{i}, \tilde{\gamma}^{i}$ are in this case real parameters. ${ }^{10}$ Let us note that under this same expansion the dual vector $\tilde{X}=\mathcal{S} X$ has respectively complex components $(-i \alpha, \ldots)$ or real ones $\left(-\tilde{\alpha}^{a}, \alpha^{a}, \ldots\right)$.

We can use expansions of different quantities in such a basis formed by charge and extra vectors, to get different results. See [1] for some illustrative cases.

\section{Concluding remarks: more about Freudenthal transformations}

The anti-involution matrix $\mathcal{S}$ can be understood as a Freudenthal duality $\tilde{q}=\mathcal{S} q[12,13]$. Under this transformation of the charges the horizon area, ADM mass and other properties of the solutions remain invariant. We have shown, for the quadratic prepotential theories studied here, that this duality can be generalized to an Abelian group of transformations ("Freudenthal transformations") of the form

$$
x \rightarrow \lambda \exp (\theta \mathcal{S}) x=a x+b \tilde{x} .
$$

Under this set of transformations applied to the charge vectors and $\mathcal{I}_{\infty}$, the horizon area, ADM mass and intercenter distances scale up, respectively, as

$$
S_{h} \rightarrow \lambda^{2} S_{h}, \quad M_{A D M} \rightarrow \lambda M_{A D M}, \quad r_{a b} \rightarrow \lambda r_{a b},
$$

\footnotetext{
${ }^{9}$ Or equivalently, $B\left(q_{n a}\right)^{\perp}$ is defined as the set of vectors $s$ such that $h(s, q)=0$ for all $q \in\left(q_{n a}\right)$, where $h$ is the Hermitian inner product defined in Section 2.

${ }^{10}$ Naturally, other bases are possible or convenient, as for example bases including linear combinations of the charge vectors, the total charge vector $Q, I_{\infty}$, etc.
} 
leaving invariant the values of the scalars at the fixed points and at infinity. In the special case $\lambda=1$, "S-rotations", the transformations leave invariant the solution. The standard Freudenthal duality can be written as the particular rotation

$$
\tilde{x}=\exp (\pi / 2 \mathcal{S}) x
$$

It is immediate to ask the question whether such transformations can be generalized to $4 d$ theories with general prepotentials, not associated to "degenerate" U-duality groups, including stringy black holes. We can see that this is indeed the case using a simple argument as follows (a more detailed investigation is presented in [17]). The U-duality quartic invariant defined ([12], using here a slightly adapted notation ) as

$$
2 \Delta_{4}(x) \equiv\langle T(x) \mid x\rangle
$$

can be written also, using the definition of Freudenthal duality, as

$$
\Delta_{4}(x)=\frac{1}{4}\langle\tilde{x} \mid x\rangle^{2}
$$

Let us note then that, for a general transformation this quantity scale as

$$
\begin{aligned}
2 \Delta_{4}(a x+b \tilde{x})^{1 / 2} & =\langle a \widetilde{x+b} \tilde{x} \mid a x+b \tilde{x}\rangle=\langle a \tilde{x}-b x \mid a x+b \tilde{x}\rangle \\
& =\left(a^{2}+b^{2}\right)\langle\tilde{x} \mid x\rangle \\
& =2\left(a^{2}+b^{2}\right) \Delta_{4}(x)^{1 / 2} .
\end{aligned}
$$

where we have used the property $a \widetilde{x+b} \tilde{x}=a \tilde{x}-b x$ This is a non trivial property as $x^{\prime}=\tilde{x}$ is a non-linear transformation, linearity is only guaranteed in the F-plane. For $a^{2}+b^{2}=1$, a $\mathcal{S}$-rotation, the quantity $\Delta_{4}$ for any $U$-duality group, and then the lowest order entropy of any extremal stringy black hole, is invariant under these transformations.

Acknowledgements. This work has been supported in part by the Ministerio de Educación y Ciencia grants FIS2011-3454, FPA2008-2356B and the Universidad de Murcia project E024-018. The work of JJ.F-M has been supported by a FPI-predoc contract FPI-2009-2132. We thank the hospitality of the CERN TH Division and of the IFT-CSIC (Madrid) where part of the research has been conducted.

\section{References}

[1] J. J. Fernandez-Melgarejo and E. Torrente-Lujan, JHEP 1405 (2014) 081 [arXiv:1310.4182 [hepth]].

[2] S. Ferrara and R. Kallosh, Phys. Rev. D 54 (1996) 1525 [hep-th/9603090].

[3] A. Ceresole, R. D’ Auria and S. Ferrara, Nucl. Phys. Proc. Suppl. 46 (1996) 67 [hep-th/9509160].

[4] S. Ferrara, R. Kallosh and A. Marrani, JHEP 1206 (2012) 074 [arXiv:1202.1290 [hep-th]].

[5] W. Israel and G. A. Wilson, J. Math. Phys. 13 (1972) 865.

[6] Z. Perjes, Phys. Rev. Lett. 27 (1971) 1668.

[7] W. A. Sabra, Mod. Phys. Lett. A 13 (1998) 239 [hep-th/9708103].

[8] P. Meessen and T. Ortin, Nucl. Phys. B 749 (2006) 291 [hep-th/0603099].

[9] J. Bellorin, P. Meessen and T. Ortin, Nucl. Phys. B 762 (2007) 229 [hep-th/0606201].

[10] M. Shmakova, Phys. Rev. D 56 (1997) 540 [hep-th/9612076].

[11] S. Ferrara, R. Kallosh and A. Strominger, Phys. Rev. D 52 (1995) 5412 [hep-th/9508072].

[12] L. Borsten, D. Dahanayake, M. J. Duff and W. Rubens, Phys. Rev. D 80 (2009) 026003 [arXiv:0903.5517 [hep-th]].

[13] S. Ferrara, A. Marrani and A. Yeranyan, Phys. Lett. B 701 (2011) 640 [arXiv:1102.4857 [hepth]]. 
[14] P. Galli, P. Meessen and T. Ortin, JHEP 1305 (2013) 011 [arXiv:1211.7296 [hep-th]]. J. J. Fernandez-Melgarejo, T. Ortin and E. Torrente-Lujan, Fortsch. Phys. 60 (2012) 1012 [arXiv:1209.3774 [hep-th]]. J. J. Fernandez-Melgarejo, T. Ortin and E. Torrente-Lujan, JHEP 1110 (2011) 068 [arXiv:1106.1760 [hep-th]].

[15] R. Kallosh and T. Ortin, JHEP 1209 (2012) 137 [arXiv:1205.4437 [hep-th]].

[16] S. Katmadas, PhD thesis. ISBN: 0471491101, 2011.

[17] J. Fernández-Melgarejo and E. Torrente-Luján, "Black holes and generalized Freudenthal transformations" to appear, . 